\title{
Research on Development Strategy of Xiaomi Mobile Phone Based on Niches Theory
}

\author{
Huixin Liu, Xiaoxiao Wang* \\ College of Management Engineering, Zhengzhou University, Science road, Zhengzhou, China \\ 630529252@qq.com,1553394250@qq.com
}

Keywords: Millet mobile phone; Ecological niche; niches width; Development strategies

\begin{abstract}
The intelligent mobile phone as the information age of fashion leader, is in the development of the fast lane. Millet mobile phone is in such a rapid development of the information age breed in the enterprise. Based on the ecological niche theory, analysis and evaluation of the resource ecological niche of millet mobile phone. This paper selected apple, HUAWEI and Jinli as a comparison of millet mobile phone who, through the computing resource niches width and niche overlap coefficient to derive the advantages and disadvantages of the mobile phone millet resources, and then find out the core competitiveness of millet mobile phone. Then according to the resource niche of millet mobile phone the results of the analysis put forward some development strategies and suggestions.
\end{abstract}

\section{Introduction}

With the development of network information technology, the Internet has set off a new generation of industrial revolution in the world (Bingxin Li, Jing-ping Liu, Yu Zhang, 2017). Some companies are beginning to look for ways to develop their own advantages in the context of the Internet, so that they can gain a place in the wave of the times. In the context of such a rapid development of the network, smart phones instead of function machines are gradually active in the historical arena. At present, mobile phone companies such as Apple, Samsung, Huawei, OPPO and vivo firmly occupy the market of smart phones. In April 2010, with the product concept of "born for fever”, Xiaomi turned out. In just a few years, Xiaomi has become a well-known national brand. The reason why Xiaomi mobile phone can develop rapidly is because it has found its core competitiveness - low price and high cost performance. However, in recent years, the competition of domestic smart phones is fierce, and the core competitiveness of Xiaomi mobile phones is constantly disintegrating. Therefore, Xiaomi needs to change its original strategy and find new advantageous resources in order to be invincible in the fierce competition.

\section{Review of related research}

\subsection{Summary of Related Research on Ecological Niche Theory}

In the field of biology, niche refers to the position of a population in an ecosystem, its position in 
time and space, and its functional relationship with related populations. Each species in the natural ecosystem has its own niche. Hutchinson proposes the concept of "basic niche", which refers to the multidimensional environmental conditions on which a population depends or grows to support a minimum number of members. The basic niche focuses on the study of individual tissue populations, and the interaction between populations is also an important part of niche theory. From an ecological point of view, if a population has a positive or negative impact on the growth of other populations, then there is an interaction between two or more populations (John Freeman, MichaelT.Hannan, 2014). If this interaction is negative, it is what we call the "competition" relationship; if the interaction is positive, it means that there is mutual or mutual benefit. Modern ecological theory defines niche as the establishment of an environmental condition in which a population can reproduce itself. This concept provides an effective way to express how environmental variables and competition affect the growth rate of a population.

\subsection{Summary of Relevant Research on Resource-Based Theory}

The first to propose resource-based theory is Wernerfelt's "Enterprise Resource Fundamentals" published in 1984. The basic idea of resource theory is to regard the enterprise as a collection of resources, focusing on the characteristics of resources and the market of strategic factors, and to explain the sustainable advantages and differences of enterprises. The resource-based theory includes three aspects: 1 . The "source" of the competitive advantage of the enterprise: special heterogeneous resources. 2. Sustainability of competitive advantage: the imitation of resources. 3 . Acquisition and management of special resources. Resource-based theory has guiding significance when companies make strategic choices. However, there are also some shortcomings. The resourcebased theory overemphasizes the internals of the enterprise and pays insufficient attention to the outside of the enterprise. Therefore, the resulting corporate strategy cannot adapt to changes in the market environment. Secondly, the determination of incomplete imitation and incomplete imitation of resources is too vague. It is very difficult to operate, and this strategic resource is also very easy to be imitated by other companies. Therefore, companies need to be cautious in the formulation of strategies, taking into account various factors.

\section{Xiaomi mobile phone resource basic analysis.}

\subsection{Company Profile}

Beijing Xiaomi Technology Co., Ltd. ("Xiaomi") is a mobile internet company specializing in the development of intelligent hardware and electronic products. "Being born with a fever" is the product concept of Xiaomi. "Let everyone enjoy the fun of technology" is the vision of Xiaomi. Xiaomi's founder and CEO Lei Jun has founded Jinshan Software, Amazon China and many other innovative companies, and its innovative spirit is also a key factor in the rapid development of Xiaomi. At the beginning of Xiaomi's founding, the main products were mobile phones, MIUI operating systems, and rice chat. Since 2013, Xiaomi has cut into the ecological chain from the field of smart home hardware and opened up group-based diversification. In addition to the three core businesses of mobile phones, TVs and routers, all Xiaomi eco-chain products belong to the "Mijia" brand. At present, Xiaomi has launched a number of offline interactive communication platforms, such as the theater-style conference, the "rice flour festival" activities, and established a number of millet homes. By the second quarter of 2017, the national sales of Xiaomi mobile phone ranked 5th. 


\subsection{Enterprise Ecological Niche Evaluation System}

The concept of niche has long been limited to the field of ecology. This paper introduces the niche theory into the strategic design of the enterprise. By placing the enterprise in the industry ecosystem, it comprehensively analyzes the internal resources and the external environment of the enterprise, clarifies the status of the enterprise at this stage, and proposes a corresponding competition strategy for the resource capability. Resource-based theory holds that companies are a collection of resources. Different enterprises have different resources for various reasons, which leads to the heterogeneity of enterprise resources. This heterogeneity determines the difference in enterprise competitiveness. The competitive advantage of the company comes from special heterogeneous resources. Therefore, the difference in resources between enterprises is an important reason for the different profitability of enterprises, and it is also the reason why enterprises with advantageous resources can obtain economic rent (Fengqiao Mei, 2016). Enterprises with special heterogeneous resources can make enterprises gain more benefits and grow up, so that they have the ability to give more quality resources. This is a gradual process. In this process, the enterprise will have energy transfer and interaction with the environment and surrounding enterprises, so its niche is also constantly developing and changing. For the analysis of niche, the paper adopts the “resource-capability-status” enterprise niche evaluation system as Shown in Figure 1.

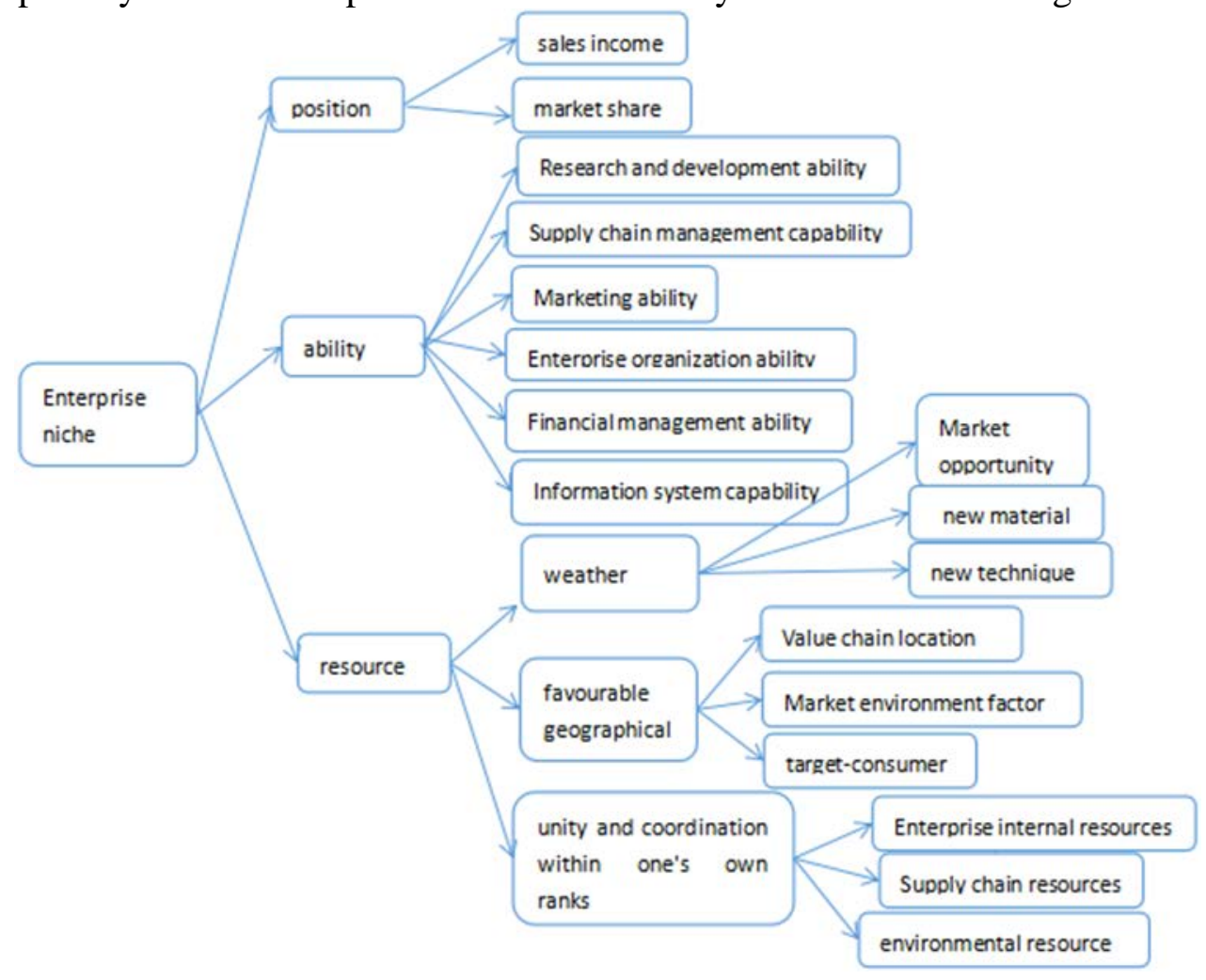

Figure 1 Enterprise niche evaluation system "Resource-competency-status".

In this paper, we mainly discuss the resource characteristics of niche. The resources in the "resource-capability-status" enterprise niche evaluation system can be analyzed from the aspects of time, location, people and three aspects. The weather and the natural weather conditions, the geographical environment and the back of the human heart. "Mencius Gongsun ugly": "Tianshi is not as good as the land, and the land is not as good as the people." "Sun Jun's Art of War·Moon War": "Heavenly, geographical, and human, the three are not allowed, although there is victory." Successful natural and socially favorable environment, timing, and conditions. Since ancient times, 
in order to succeed in one thing, it is often necessary to rely on the three conditions of "time, place, and people." Similarly, if a company wants to succeed, it needs to rely on "time, place, and people" to adapt to the opportunity, find the right direction, and make full use of its superior resources in order to obtain sustainable development conditions.

\subsection{Enterprise Ecological Niche Overlap Measure}

\subsubsection{Niche Width and Niche Overlap}

Biologists characterize niche by using a series of indicators such as niche width and niche overlap. The niche width, also known as niche breadth or niche size, is the sum of various resources that a species can use. If a population has a positive or negative impact on the growth of other populations, then there is an interaction between two or more populations. When considering the interaction between populations, the growth of one of the populations will change the niche breadth of the other populations. Analogous to the breadth of biological niche, the niche breadth of enterprises refers to the sum of various market resources utilized by enterprises, that is, the degree of diversification of market environment resources. Generally speaking, the wider the niche, the stronger the adaptability of the enterprise to the environment; on the contrary, the weaker the adaptability of the enterprise, once the market environment changes, the enterprise can not respond in time, it will be at risk. If the geographical location, time of establishment, and various resources required by the company are similar, then the niche of the enterprise will overlap.

Ecologists often define niche overlap as the common use of dissimilar species for specific resource locations. The methods for measuring the niche breadth and niche overlap are mainly Levins formula, Hurlbert formula, Pianka formula, and Pe-traitis formula. Because each method has certain advantages and disadvantages, it can be selected according to the actual situation in the actual research (Ligang Gu, Ying Lan, Shelley, 2016).

\subsubsection{Calculation Method of Ecological Niche Width Overlap Degree}

This paper will measure the niche overlap from the perspective of niche width. For the i-th enterprise, the occupied width on the market resource dimension $\mathrm{p}$ is represented by Lip, and the overlap width of the enterprise $i$ and the enterprise $j$ on $p$ is set to li, $j, p$, then the enterprise $i$ and the enterprise $\mathrm{j}$ are on the $\mathrm{p}$ The niche overlap coefficient of the market resources enterprise is (Xingyuan Wang, 2006):

$$
\mathrm{O}_{\mathrm{ij}}=\mathrm{l}_{\mathrm{i}, \mathrm{j}, \mathrm{p}} / \mathrm{L}_{\mathrm{ip}}
$$

The range is: $0 \leq{ }^{O_{i j}} \leq 1$. Equation (1) is an asymmetry formula. If two companies have the same degree of utilization of a certain resource, the degree of overlap calculated by (1) is equivalent; on the contrary, if the occupation of the same resource by two companies is assumed to be extreme, One enterprise is very similar to a monopoly, and the other is extremely small at 0 . At this time, their overlap has obvious effects on the two.

\subsection{Mobile Phone Enterprise Resource Ecological Niche Evaluation}

\subsubsection{Selection of Research Objects}

According to the well-known market research firm Strategy Analytics released in the second quarter of 2017, global smartphone manufacturers' shipments and market share reports show that Samsung ranks first in the world with 79.5 million shipments. Among TOP5 manufacturers, there 
are three brands of domestic mobile phones. On the list. Apple shipped 41 million units, with a market share of $11.4 \%$, ranking second, down 0.4 percentage points year-on-year. With the release of the iPhone 8 in the third quarter, Apple's market share is expected to increase further. The top three mobile phones in China, Huawei, OPPO and Xiaomi ranked 345. Huawei shipped 38.4 million units in the second quarter, ranking first in domestic mobile phones, and its market share increased from $9.4 \%$ in the same period last year to $10.7 \%$. OPPO has the largest year-on-year growth, with shipments reaching 29.5 million units. The market has come to 8.2\%, an increase of 2.9 percentage points over the same period last year. Xiaomi has also improved significantly, with shipments of 23.2 million units and a market share of $6.4 \%$, returning to the top five in the world. In the Internet era, Xiaomi mobile phone has developed rapidly since its establishment and has achieved amazing results. Its success has also become the industry's advertised. As a veteran of domestic brands, Huawei mobile phones have been leading the sales in recent years.Since the prelude of Apple's mobile phone to open the smart phone, Apple has been in the leading position in the smart phone industry with its own resource advantages. Although the Jinli mobile phone is ns not as good as the Xiaomi mobile phone, it is also experiencing a rapid rise. This paper selects Xiaomi mobile phone as the target enterprise, Apple and Huawei as the comparison enterprises, and Jinli as the comparison enterprise, and the four are making evaluations on the basis of mobile phone resources.

\subsubsection{Mobile Phone Enterprise Resource Niche Evaluation Design}

On the basis of the "resource-capability-status" enterprise niche evaluation system, this paper focuses on the evaluation of mobile phone enterprises from the "resources" layer. The indicators in the resource layer time, space and environment will be based on the resource characteristics of mobile phone enterprise services. Refine (Huixin Liu, Xingchen Liu, 2015).

(1)Indicators and weight settings

1) Time resource. The release of the iphone 4 mobile phone in June 2010 marked the entry of the global mobile phone industry into the era of smart phones. The update of smartphones is very fast, and the competition in the mobile phone industry is also increasing. It is therefore important to innovate at all times to meet the growing needs of the people. This paper divides time resources into technological advancement, advanced parts and market leading capabilities. The main competitiveness of the mobile phone industry is more important in terms of technological advancement. The setting of indicators is mainly analyzed from important hardware aspects. Market leadership can be analyzed in terms of when it is established and how fast it is updated.

2) Space resources. This paper describes spatial resources from three aspects: market location, consumer location, and value chain location. Market location is based on the fit of product and market, and it is divided into three aspects: customer recognition, market size and consumer purchasing power; consumer location is divided into price range and product series; marketing and marketing at both ends of smartphone value chain Design is a value-added link, and the main intermediate supply chain links are less value-added, so the weight of the value chain location is set smaller. Since the use of space resources by smartphones mainly depends on market location and consumer location, the weights of these two types are set larger.

3) Environmental resources. The environmental resource is "human harmony", which mainly considers three aspects of enterprise scale, supply chain coordination and environmental coordination. The scale of the enterprise is measured by the total assets; the coordination of the supply chain can be subdivided into operating system platforms, CPU suppliers and sales channels; environmental coordination is the relationship between Chinese policies and corporate development.

The specific model is shown in figure 2. 


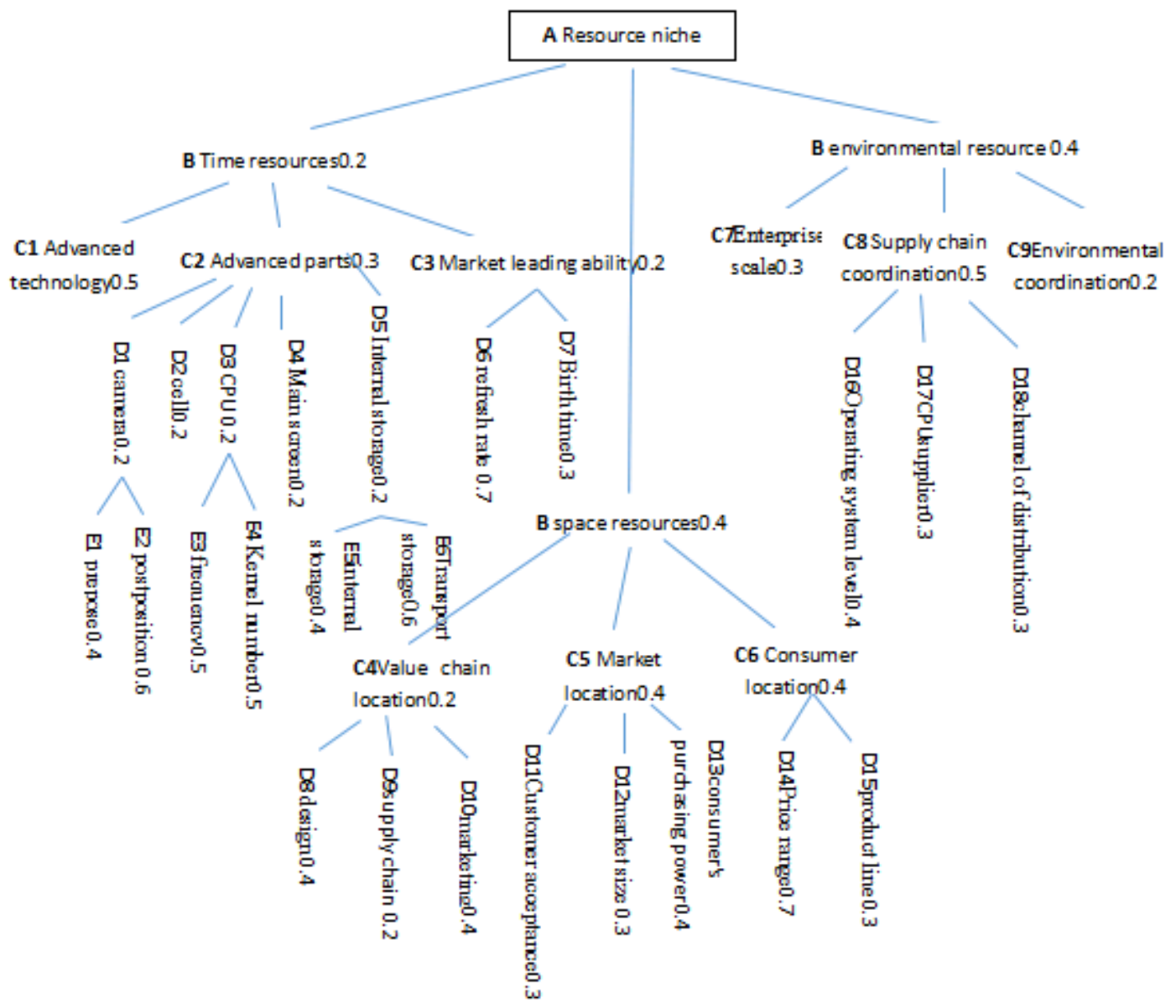

Figure 2 Mobile phone enterprise resource niches analysis framework.

(2) Indicator classification and assignment description

The mobile phone enterprise resource framework proposed in this paper is divided into A, B, C, $\mathrm{D}$, and E5 layers, and the degree of subdivision is increased from top to bottom. As shown in Figure 2. These indicators are divided into three categories.

1) Data quantification indicators. The final layer indicators (E1-E6, D2, D4, D6, D7, D14, D15) subdivided under C2, C3, and C6, a total of 12 indicators. These metrics require data support.

Apple's latest PhoneX rear camera is 12 million pixels. The rear camera of Huawei Mate10 mobile phone is 12 million pixels plus 20 million pixels. Therefore, on the E2 indicator, the score of the Apple mobile phone is lower than that of the Huawei mobile phone.

2) Measure the quantitative indicators. The final layer indicators (D13-D15), C7, and C9 under the $\mathrm{C} 1$ and $\mathrm{C} 5$ subdivisions have a total of six indicators. These indicators have no way to conduct detailed assessments and can be scored based on actual conditions. Although this method of scoring is not subjectively accurate, it reflects to some extent the extent to which the mobile phone industry utilizes designated resources.

3) 0-1 quantitative indicators. The final layer indicators (ie D8-810, D16-D18) subdivided under C4 and C8, a total of 6 indicators. Such indicators tend to be "yes" or "no" when analyzing individual resources. For example, when analyzing the specific links under the value chain location, the first thing to focus on is whether the company occupies this link, and then analyze how good or bad it is in this link. 
(3)Frame assignment and result analysis

According to the data query of the above four mobile phone companies, including the comparison of text and data, the value of "the degree of resource utilization" is assigned, ranging from $0-10$, as shown in the following table 1.

Table 1 Mobile enterprise resource niche framework final layer assignment.

\begin{tabular}{|c|c|c|c|c|c|c|c|c|c|}
\hline & apple & huawei & xiaomi & Jinli & & apple & huawei & Xiaomi & Jinli \\
\hline C1 & 10 & 8 & 7 & 4 & D12 & 7 & 8 & 9 & 3 \\
\hline C7 & 9 & 6 & 5 & 3 & D13 & 9 & 8 & 7 & 3 \\
\hline C9 & 7 & 10 & 9 & 7 & D14 & 10 & 7 & 6 & 8 \\
\hline D2 & 5 & 9 & 6 & 8 & D15 & 9 & 5 & 7 & 4 \\
\hline D4 & 6 & 7 & 6 & 7 & D16 & 10 & 8 & 9 & 5 \\
\hline D6 & 7 & 7 & 8 & 3 & D17 & 8 & 6 & 5 & 3 \\
\hline D7 & 10 & 7 & 3 & 8 & D18 & 6 & 8 & 9 & 4 \\
\hline D8 & 9 & 7 & 8 & 4 & E1 & 8 & 9 & 10 & 7 \\
\hline D9 & 0 & 3 & 7 & 3 & E2 & 7 & 8 & 10 & 8 \\
\hline D10 & 10 & 6 & 8 & 4 & E3 & 8 & 10 & 6 & 10 \\
\hline D11 & 10 & 9 & 8 & 5 & E4 & 8 & 6 & 9 & 4 \\
\hline D12 & 7 & 8 & 9 & 3 & E5 & 7 & 9 & 8 & 8 \\
\hline D13 & 9 & 8 & 7 & 3 & E6 & 7 & 7 & 9 & 9 \\
\hline
\end{tabular}

Combined with figure 2 and Table 1, calculate from right to left, layer by layer. Finally, the relationship between time resources, space resources, environmental resources and enterprise niche is obtained, as shown in Table 2.

Table 2 Results of niche assessment of smartphone resources.

\begin{tabular}{|l|l|l|l|l|}
\hline & apple & Huawei & Xiaomi & Gionee \\
\hline Time resources (days) & 8.58 & 8.01 & 7.17 & 5.19 \\
\hline Space resources (geographical location) & 8.68 & 7.04 & 7.24 & 4.92 \\
\hline $\begin{array}{l}\text { Environmental resources (human } \\
\text { harmony) }\end{array}$ & 8.2 & 7.5 & 7.2 & 4.35 \\
\hline Total resource position & 8.468 & 7.418 & 7.21 & 4.746 \\
\hline
\end{tabular}

Comparing Table 1 and Table 2, there is still a certain gap between Xiaomi mobile phone and Apple mobile phone in terms of "time, location, and people". Compared with Huawei's mobile phones, Xiaomi's mobile phones have advantages in terms of "land advantage" and relatively poor in "daytime, people and harmony". Compared with the Jinli mobile phone, Xiaomi mobile phone has great advantages in all aspects, but the advantage in "Tianshi" is not obvious. Therefore, Xiaomi mobile phone should strengthen the use of time resources and environmental resources, but to clarify the degree of utilization and expansion space of target enterprises in individual resources, it is necessary to further analyze the overlapping of resources.

(4)Resource niche overlap calculation

According to the specific subdivision of the smart phone enterprise resource evaluation indicators shown in Figure 2, combined with the collected resource data summary, calculate and define the superior resources of the above enterprises from an overlapping perspective. Because the final layer indicators are numerous and cumbersome, in order to simplify the calculation, this paper only calculates the C-level indicators. Calculated according to Figure 2 and Table 1, the calculation results are shown in the table below. 
Table 3 Niche overlap factor for C layer indicators of smart phones.

\begin{tabular}{|l|l|l|l|l|l|l|l|l|}
\hline & C1 & C2 & C4 & C5 & C6 & C7 & C8 & C9 \\
\hline Oxi & 0.7 & 0.87 & 1 & 0.91 & 0.65 & 0.56 & 0.95 & 0.78 \\
\hline Oxh & 0.87 & 0.94 & 0.74 & 0.95 & 1 & 0.83 & 0.95 & 0.90 \\
\hline Oxj & 0.57 & 1 & 0.49 & 0.46 & 0.93 & 0.60 & 0.53 & 0.78 \\
\hline
\end{tabular}

\subsubsection{Analysis}

Combined observations and analysis of Table 4 lead to the following conclusions.

(1)Compared with Jinli mobile phone, Xiaomi mobile phone has an advantage in 7 indicators. Among them, in terms of technological advancement, value chain location, market location and supply chain coordination, Xiaomi mobile phone has an absolute advantage. Jinli mobile phone has been established as early as 2002. With the mission of "Gold Quality, Establishing the World", it pays attention to the requirements of excellent quality. This also makes the use of Gionee mobile phones more successful people, and the price is higher. Xiaomi mobile phone is a product of the Internet era, and it closely follows the footsteps of the times and continues to innovate. Xiaomi mobile phone is also the only mobile operating system-MIUI system with independent research and development in China, which makes Xiaomi mobile phone better in technology and value chain. Xiaomi mobile phone is cost-effective, mainly for the middle and low-end people, so it has great advantages in terms of market location.

(2) Apple mobile phones and Huawei mobile phones ranked higher in overall sales than Xiaomi. The main reason is that there are disadvantages in market leading ability, consumer location, enterprise scale and market location. In the smart phone industry, Apple's mobile phone is a welldeserved leader, and Xiaomi mobile phone has always been Apple's follower. Established in 2010, Xiaomi Mobile has grown rapidly in just seven years. With the "Hunger Marketing", social platform marketing and high cost performance, it has quickly become one of the top five in the country. However, with the fierce competition in online marketing, Xiaomi's advantage is no longer, and instead of the offline sales channel - Xiaomi's home, there is no way to take care of Xiaomi's mobile phone. Therefore, compared with the relatively mature Huawei and Apple, Xiaomi has relatively few resources available in the market location and consumer location. In recent years, many mobile phone industries are imitating Xiaomi's products and sales methods. Xiaomi mobile phones need to continue to innovate in order to form the core competitiveness of products (Xiangyu Ji, Qian Wa)ng, 2017.

To sum up, the resources corresponding to the index whose niche overlap coefficient is not greater than 0.9 are regarded as the dominant resources in this paper. Therefore, the advantage resources of Xiaomi mobile phone are C1 technology advancement, C4 value chain location, C7 enterprise scale and C9 environment coordination.

\subsection{Strategy Recommendations for Xiaomi Handsets}

(1) Improve brand value. Since its establishment, Xiaomi Mobile has gradually achieved worldrenowned achievements with the help of the advantages of the Internet. However, since its inception, Xiaomi has been Apple's most loyal follower. Xiaomi CEO Lei Jun was dubbed "Rebs", in order to maintain coexistence, choose distance to follow and choose to follow the strategy. The application of Xiaomi to this strategy is perfectly differentiated by "low price and high cost performance". In recent years, other mobile phone companies have been eager to follow the success of Xiaomi. Xiaomi's proud low-cost strategy is unsustainable. Xiaomi's top priority is to learn from the dynamic zone and start from the brand's four-edge model-product, value, symbol and positioning. 
The curse increases the value of its own brand and enhances its defense ability (Qiuyuan Dong, 2017).

(2) The brand positioning is accurate. "Born for a fever" is the product concept of Xiaomi, and it is also the brand positioning that Xiaomi insisted on at the beginning. Today's Xiaomi is no longer a dedicated custom product that "fans" can be proud of. The low price helped Xiaomi quickly open up the smart machine market, but it also made its target group far beyond the envisioned "mobile phone enthusiasts" and became a low-end consumer group based on low prices. If the original millet uses system optimization and hardware upgrades to attract consumers, today's Xiaomi has been unable to do anything about its technological innovation capabilities. Cultivate customer stickiness, upgrade consumer focus and purchase behavior from price stickiness to brand stickiness, and cultivate competitive customer loyalty. Therefore, Xiaomi should strengthen technological innovation and identify the brand positioning.

(3) Create core technologies. Since its inception, Xiaomi Mobile has been designed to enable consumers to use high-priced and reliable electronic products. In recent years, Xiaomi Mobile has built its core technological advantages to compete with the industry. Xiaomi mobile phone first used Qualcomm Xiaolong flagship processor, dual-core 1.5GHZ processor was a very high-end configuration at the time, the same configuration of other brands of mobile phones are more than 3,000 yuan. Since the birth of the Snapdragon 800 series, Xiaomi has accounted for 66\% of Qualcomm China's shipments, and other domestic brands add up to 34\%. After all, China is the world's largest consumer of smart phones, and its shipments in China are worth more than others. Sales in many countries. In addition, Xiaomi also spent a lot of effort on the screen, set up a team dedicated to the screen of the core device department, in order to reduce the blue light damage caused by the mobile phone, and has a high-intensity sunlight screen, developed a 1nit ultra-dark night screen, Playing mobile phones at night is not glaring. In the future development of Xiaomi mobile phone, we can make persistent efforts in research and development technology to create core technology to produce cost-effective smart phones and form our own new competitive advantages.

\section{Conclusions}

Xiaomi has already entered the mobile phone industry with a subversive attitude. Not only has it achieved impressive results, but it has also forced the upgrading of the entire industry. The ecological chain enterprises invested by Xiaomi Company also play the role of the industry disruptor by Xiaofeng's Dongfeng. It can be said that the millet product is linked to the explosion. The success of Xiaomi is also the center of the public opinion of Xiaomi. After experiencing "patent problems", quality door events and false publicity events, Xiaomi's mobile phone sales are not as good as before. In this paper, the theory of niche is applied to the research of resources of Xiaomi mobile phone, and the resource framework is constructed through three aspects: time resource (day time), space resource (location) and environmental resource (human and), and the niche breadth and ecology are utilized. The overlap degree calculates the niche overlap coefficient, and finally analyzes the advantages and disadvantages of the Xiaomi mobile phone and the direction of further efforts. In the contemporary competition of smart phones, Xiaomi mobile phones should give full play to their own advantages, turn disadvantages into advantages, continue to innovate, and find and develop new core competitiveness. 


\section{References}

[1] Bingxin, Li, Jing-ping, Liu, Yu, Zhang, 2017. Research on the Mode and Mechanism of customer participation in Brand value creation under Network Environment-taking Xiaomi Mobile phone as an example. Journal of Beijing Industrial and Commercial University. 32(1), 61-72.

[2] Fengqiao, Mei, 2016. Development Strategy of Ecological Civilization in Smart phone Industry. Science and Technology Predictions. 34(09), 69-70.

[3] John, Freeman, MichaelT.Hannan, 2014. Tissue Ecology. Science Press.Shanghai, 1nd edition..

[4] Huixin, Liu, Xingchen, Liu, 2015. Enterprise Resource Analysis Based on the "Resource-Capacity-Status" Niche Evaluation System.Enterprise Economy. (01), 11-16.

[5] Ligang, Gu, Ying, Lan, Shelley, 2016. Study on the Stability of Business Ecosystem from the Perspective of Enterprise Niche. Industrial Economy and Technology. 35(05), 148-155.

[6] Qiuyuan, Dong, 2017. From the "fan economy" to the transformation of the brand community-based on the decline in Xiaomi mobile phone sales. Modern commerce and industry. 38(11), 41-43.

[7] Xingyuan, Wang, 2006. A study on niche overlap Measurement Model of the same Brand Market. China Market Association 2006 Annual meeting and the fourth National Congress of the members of the paper.

[8] Xiangyu, Ji, Qian, Wang, 2017. The growing pains of Xiaomi. Business management.(08), 43-46. 